\title{
Aspectos sociodemográficos e clínicoepidemiológicos da tuberculose em um município do nordeste brasileiro
}

\author{
Sociodemographic, clinical and epidemiological aspects of tuberculosis in a brazilian \\ northeastern municipality
}

Aspectos sociodemográficos y clinicoepidemiológicos de la tuberculosis en un municipio nordestino brasileño

Pedro Henrique dos Santos Silva ${ }^{1 *}$, Sara Sabrina Vieira Cirilo², Severino Cavalcante de Sousa Junior $^{1}$, Victor Trindade da Cruz ${ }^{1}$, Rafael Santos Correia ${ }^{1}$ Andréia Ferreira dos Santos ${ }^{1}$, Tom Ravelly Mesquita Costa ${ }^{1}$, Carolina Gomes Vieira ${ }^{1}$, Andréia Santos Miranda ${ }^{1}$.

\section{RESUMO}

Objetivo: Realizar análise epidemiológica da correlação entre fatores sociodemográficos e a incidência da tuberculose. Métodos: Trata-se de um levantamento epidemiológico descritivo desenvolvido em um município do nordeste brasileiro sobre a incidência da tuberculose e a sua relação com os fatores sociodemográficos. A análise dos dados objetivou promover a análise da correlação entre o surgimento de novos casos de tuberculose e as variáveis sexo, raça, zona de moradia e faixa etária das pessoas acometidas. Resultados: Observou-se que a incidência da tuberculose apresenta diferenças estatísticas significativas em relação a todos os parâmetros analisados, acometendo mais homens $(63,2 \%)$, pardos $(60,2 \%)$, habitantes da zona urbana (88,4\%), e com faixa etária entre 20 e 64 anos (70,6\%). Conclusão: O presente estudo identificou que as variáveis analisadas na população do município estão diretamente associadas à incidência da tuberculose, demonstrando a correlação entre elas. A ocorrência da tuberculose deve ser entendida em seus vários aspectos, transpassando a visão clínica, para o entendimento dos fatores sociais que contribuem com a sua incidência, o que torna possível ações direcionadas de controle.

Palavras-chave: Tuberculose, Epidemiologia, Saúde pública, Fatores socioeconômicos.

\begin{abstract}
Objective: To perform a correlation epidemiological analysis between sociodemographic factors and the incidence of tuberculosis. Methods: This is a descriptive epidemiological survey developed in a city in Northeaster Brazil on the incidence of tuberculosis and its relationship with sociodemographic factors. The data analysis aimed to promote the analysis of the correlation between the emergence of new cases of tuberculosis and the variables gender, race, area of residence and age group of affected people. Results: It was observed that the incidence of tuberculosis presents significant statistical differences in relation to all analysed parameters, affecting more men $(63.2 \%)$, browns $(60.2 \%)$, urban dwellers $(88.4 \%)$ and aged between 20 and 64 years $(70.6 \%)$. Conclusion: The present study identified that the variables analysed in the municipality population are directly associated with the incidence of tuberculosis, demonstrating the correlation between them. The occurrence of tuberculosis must be understood in its various aspects, going beyond the clinical view, to the understanding of the social factors that contribute to its incidence, which makes possible directed control actions.
\end{abstract}

Key words: Tuberculosis, Epidemiology, Public health, Socioeconomic factors.

${ }^{1}$ Universidade Federal Do Piauí (UFPI), Parnaíba - PI. *E-mail: ph_beta@homail.com

2 Universidade Estadual Do Maranhão (UEMA), Caxias - MA. 


\section{RESUMEN}

Objetivo: Realizar un examen epidemiológico de la correlación entre los factores sociodemográficos y la incidencia de la tuberculosis. Métodos: Esta es una encuesta epidemiológica descriptiva desarrollada en una ciudad del noreste del Brasil sobre la incidencia de la tuberculosis y su relación con los factores sociodemográficos. El análisis de los datos tuvo como objetivo promover el análisis de la correlación entre la aparición de nuevos casos de la tuberculosis y las variables género, raza, área de residencia y grupo de edad de las personas afectadas. Resultados: Se observó que la incidencia de la tuberculosis presenta diferencias estadísticas significativas en relación con todos los parámetros analizados, afectando a más los hombres (63.2\%), los marrones (60.2\%), habitantes urbanos (88.4\%) y con edades comprendidas entre 20 y 64 años $(70,6 \%)$. Conclusión: El presente estudio identificó que las variables analizadas en la población del municipio están directamente asociadas con la incidencia de tuberculosis, lo que demuestra la correlación entre ellas. La aparición de la tuberculosis debe entenderse en sus diversos aspectos, más allá de la visión clínica, para comprender los factores sociales que contribuyen a su incidencia, lo que hace posible las acciones del control dirigido.

Palabras clave: Tuberculosis, Epidemiología, Salud pública, Factores socioeconómicos.

\section{INTRODUÇÃO}

A tuberculose (TB) é uma doença infectocontagiosa, e uma das doenças mais antigas conhecidas pela humanidade, com registros históricos encontrados em múmias egípcias, em antigos escritos chineses, em estudos de Hipócrates na Grécia antiga e em trechos de livros hebraicos bíblicos (KOZAKEVICH GV e SILVA RM, 2015). Seu agente etiológico é o bacilo Mycobacterium tuberculosis, também conhecido como bacilo de Koch (BK). A infecção por essa bactéria atinge principalmente os pulmões, causando a tuberculose pulmonar, podendo, entretanto, atingir também diversas outras partes do corpo, causando, assim, o desenvolvimento da tuberculose extrapulmonar (SILVA MEN, et al., 2018; MINISTÉRIO DA SAÚDE, 2011).

A transmissão da tuberculose se dá habitualmente de pessoa para pessoa, por meio da inalação de gotículas expelidas pela tosse, espirro ou fala de uma pessoa doente com TB ativa na forma pulmonar ou, mais raramente, laríngea (PROCÓPIO MJ, 2014; MINISTÉRIO DA SAÚDE, 2017). Caso ocorra a inalação da partícula infectante, esses indivíduos passam a ser chamados de infectados, e desses, estima-se que 10\% desenvolverão a doença (PROCÓPIO MJ, 2014).

Diversos fatores são responsáveis por potencializar a capacidade de transmissão da bactéria por via inalatória. A intensidade de contato com indivíduos doentes, em relação a proximidade, tempo e local onde ele ocorre, é um importante fator de risco para infecção, visto que ambientes fechados e com grande fluxo de pessoas facilitam muito o processo de transmissão (KOZAKEVICH GV e SILVA RM, 2015). Algumas variáveis ambientais podem reduzir as probabilidades de que as partículas infectantes sejam inaladas, como as correntes de ar que dispersam as partículas no ambiente, a luz ultravioleta e a radiação gama provenientes do sol, que inativam os bacilos (PROCÓPIO MJ, 2014).

A principal localização da infecção pelo bacilo de Koch é a pulmonar, e os sinais e sintomas típicos da manifestação da tuberculose decorrem das respostas do sistema imune do hospedeiro à presença dos antígenos (MINISTÉRIO DA SAÚDE, 2011). Em sua forma pulmonar, o sintoma mais frequente é a tosse, que pode ser inicialmente seca, e posteriormente produtiva, com expectoração mucoide ou purulenta, sendo as vezes acompanhada por sangue, sintoma que geralmente dura mais que três semanas (MINISTÉRIO DA SAÚDE, 2013).

Além da tosse, a tuberculose se manifesta como uma síndrome infecciosa crônica, com febre baixa, consumindo o doente, causando debilidade e emagrecimento, sintomas clássicos da doença. Além da forma pulmonar, sabe-se que a TB também pode se desenvolver em outros órgãos do corpo humano, onde a forma mais prevalente é a pleural, que se manifesta com a ocorrência aguda de dor torácica e febre, podendo ser confundida com quadro de pneumonia (PROCÓPIO MJ, 2014).

REAS/EJCH | Vol.12(5) | e1916 | DOI: https://doi.org/10.25248/reas.e1916.2020 Página 2 de 10 
Alguns grupos populacionais têm uma maior suscetibilidade à infecção pelo BK. As pessoas portadoras do Vírus da Imunodeficiência Humana (HIV), os tabagistas, os usuários de drogas ilícitas, os alcóolatras, os diabéticos, os idosos e as pessoas em condições que causam imunossupressão, como os que fazem tratamento de câncer com quimioterápicos, têm maior probabilidade de adoecer (RABAHI MF, 2012; CUNHA $\mathrm{EA}$, et al., 2018). Além desses fatores, diversos determinantes sociais contribuem para a manutenção da tuberculose como um problema de saúde pública. Situações de pobreza extrema, ocorrência desnutrição, oferta de más condições sanitárias e a alta densidade populacional, características comuns em países em desenvolvimento, estão diretamente relacionadas com a alta ocorrência da TB nesses locais (PEREIRA AG, et al., 2018).

Desse modo, o diagnóstico precoce da TB é essencial, e se mostra como o primeiro passo para que haja controle da doença (MELLO FCQ, 2018). A confirmação do diagnóstico se dá principalmente com a realização da baciloscopia pelo método Ziehl-Neelsen, um exame simples, rápido e econômico (PROCÓPIO MJ, 2014; CAMPOS HS, 2006).

Apesar de todos os esforços para controle, atualmente a tuberculose ainda persiste como um importante problema de saúde. Segundo a Organização Mundial da Saúde (OMS), a TB foi responsável por aproximadamente 1,5 milhões de mortes em todo o mundo, no mesmo ano (SILVA DR, et al, 2018; SANTOS JN, et al, 2018). Somente no Brasil, foram notificados 72.770 novos casos de tuberculose no ano de 2017 (MINISTÉRIO DA SAÚDE, 2018; FERREIRA ALS, et al., 2018; PELISSARI DM, et al., 2018).

O tratamento da TB, realizado de acordo com as recomendações do Ministério da Saúde, inclui as fases intensiva e de manutenção. A fase intensiva tem a função de reduzir rapidamente a população bacteriana e eliminar as que possuem resistência a algum medicamento, o que tem como principal consequência a redução da contagiosidade. Já a fase de manutenção, tem como objetivo eliminar as bactérias latentes e reduzir a possibilidade de recidiva (MINISTÉRIO DA SAÚDE, 2018). Entretanto, ainda são encontradas muitas dificuldades na montagem serviço eficiente de notificação e controle epidemiológico. Estima-se que, no Brasil, $39,4 \%$ dos casos de TB não sejam notificados ao Sistema de Informação de Agravos de Notificação (SINAN) (OLIVEIRA GP, et al., 2012).

O abandono do tratamento é um dos maiores empecilhos ao controle da TB no Brasil. Em 2016, o percentual de sucesso de tratamento para os casos novos com confirmação laboratorial foi de $74,6 \%$, com $10,8 \%$ de abandono de tratamento, e $4,1 \%$ dos registros com informação ignorada quanto ao desfecho (MINISTÉRIO DA SAÚDE, 2018). Nesse contexto, visando uma modificação da realidade encontrada, é necessário que as Equipes de Saúde da Família (ESF) atuem abordando as pessoas infectadas, ou com suspeita de infecção, para que seja realizado o correto encaminhamento e acompanhamento dos casos confirmados (SANTOS TA e MARTINS MMF, 2018; AZEVEDO MAJ, et al., 2018; RODRIGUES FF, et al., 2018).

Desse modo, a ocorrência da TB deve ser entendida em seus vários aspectos, transpassando a visão clínica, de modo a promover um entendimento completo não só dos seus aspectos biológicos, mas principalmente dos fatores sociais que estão em sua origem e dificultam o seu combate, para, dessa forma, revelar populações e regiões mais vulneráveis. O objetivo deste trabalho foi realizar a análise epidemiológica da correlação entre fatores sociodemográficos e a incidência da tuberculose.

\section{MÉTODOS}

Trata-se de um estudo epidemiológico quantitativo descritivo desenvolvido sobre a incidência da tuberculose e a sua correlação com os fatores sociodemográficos da população acometida. O levantamento tomou por base os dados epidemiológicos armazenados pela Prefeitura da cidade referentes ao período de 2001 a 2016. Para a realização desse trabalho, foram analisados os dados referentes ao sexo, à raça, ao local de moradia, e à faixa etária dos pacientes. Essas informações foram obtidas através da análise das fichas de investigação emitidas pelas unidades de saúde do município, e encaminhadas para o Sistema de Informação de Agravos de Notificação. 
O município se localiza no litoral do estado do Piauí. Segundo o último censo realizado pelo Instituto Brasileiro de Geografia e Estatística (IBGE) no ano de 2010, sua população era de 145.405 pessoas, com uma concentração de aproximadamente $94,49 \%$ morando na zona urbana da cidade (IBGE, 2019). O Produto Interno Bruto per capita do município era de $\mathrm{R} \$ 12.787,32$, e a renda mensal média girava em torno de 1,7 salários mínimos. A cobertura de esgotamento sanitário era na faixa de $23,5 \%$. Quanto à rede de saúde do município, existem 16 Unidades Básicas de Saúde (UBS), com atuação dividida em 21 módulos. Como centro de referência em cuidado para os pacientes com tuberculose existe um hospital especialista (IBGE, 2019).

Para a realização desse estudo, foram utilizados apenas os casos incidentes, representados pelos casos novos de TB notificados no município. Os dados obtidos foram tabelados usando o programa Microsoft Office Excel $2013 \AA$, analisados e comparados por métodos estatísticos paramétricos.

Esse estudo foi submetido e aprovado pelo Comitê de Ética em Pesquisa da Secretaria Municipal de Saúde, e protocolado sob o número 1005/2015. A utilização das bases de dados foi autorizada pelo gestor responsável e foi garantido o sigilo dos dados de identificação dos pacientes.

Os dados foram analisados, primeiramente pelo teste de homogeneidade de variância do resíduo, e em seguida foi realizada uma análise quantitativa de variância, do programa estatístico Statistical Analysis System, (2003), versão 9.2.2. Foi aplicado o teste de Tukey à $5 \%$ de probabilidade para as comparações de médias (SAS, 2003).

\section{RESULTADOS}

O levantamento de dados realizado pela Prefeitura municipal, sobre o perfil epidemiológico da cidade, em relação à incidência da tuberculose, na população utilizou como base os dados coletados nas Unidades Básicas de Saúde, por meio do atendimento aos pacientes feito pelos médicos, utilizando fichas de anamnese, durante o período de 2001 a 2016 e notificados ao SINAN. A partir dele, pode-se avaliar a influência dos fatores sociodemográficos com a ocorrência de novos casos de tuberculose.

A Tabela 1 mostra os números totais dos casos de TB notificados no município, demonstrando uma média de aproximadamente 71,6 casos por ano. Além disso, nos últimos anos notou-se uma tendência a redução do número de novos casos, passando de uma média de 74,4 casos anuais na década passada, para 67 casos anuais de média nessa década. Com relação à delimitação dos casos por sexo do paciente acometido por TB, o presente estudo observou maior incidência em indivíduos do sexo masculino, com média de 45,31 casos por ano, que do sexo feminino, com uma média de 26,31 casos por ano, como mostra a tabela 1. Demonstrou-se uma maior prevalência da doença em pacientes da cor parda, grupo que, excluindo-se os casos notificados em que o parâmetro da raça não foi considerado, representa $72 \%$ do total de pacientes.

A Tabela 2 mostra a distribuição dos casos de TB de acordo com o local de moradia do paciente. A partir dela, observa-se que a grande maioria dos casos de TB é proveniente da zona urbana, representando aproximadamente $88,4 \%$ dos casos notificados, excluindo-se aqueles em que esse parâmetro não foi investigado. A Tabela 2 mostra também uma incidência muito maior da doença em pacientes com idade entre 20 e 34 anos, diferindo estatisticamente das demais faixas de idade. Esse intervalo, entre 20 e 34 anos de idade, representa a maior incidência, com aproximadamente $30 \%$ do total de casos no período analisado, e uma média de incidência de 21,93 casos anuais da doença. Com relação a esse fator, observou-se que não houve notificação no ano de 2001, sendo, portanto, considerado apenas o período de 2002 a 2016. 


\section{Revista Eletrônica Acervo Saúde / Electronic Journal Collection Health ｜ ISSN 2178-2091}

Tabela 1 - Distribuição dos casos de Tuberculose em um município do estado do Piauí classificados por sexo e raça.

\begin{tabular}{|c|c|c|c|c|c|c|c|c|c|}
\hline \multirow[b]{2}{*}{ Variáveis } & \multicolumn{2}{|c|}{ Sexo } & \multicolumn{5}{|c|}{ Raça/Cor da pele } & \multirow[b]{2}{*}{$\begin{array}{l}\text { Número de } \\
\text { casos de } \\
\text { tuberculose }\end{array}$} & \multirow[b]{2}{*}{$\begin{array}{c}\text { Porcentagem } \\
\text { anual }\end{array}$} \\
\hline & Masculino & Feminino & $\begin{array}{c}\text { Ignorado/Em } \\
\text { Branco }\end{array}$ & Branco & Preta & Amarelo & Pardo & & \\
\hline 2001 & 47 & 27 & 74 & 0 & 0 & 0 & 0 & 74 & $6,4 \%$ \\
\hline 2002 & 35 & 25 & 59 & 1 & 0 & 0 & 0 & 60 & $5,2 \%$ \\
\hline 2003 & 49 & 23 & 31 & 0 & 1 & 4 & 36 & 72 & $6,2 \%$ \\
\hline 2004 & 40 & 19 & 0 & 1 & 2 & 0 & 56 & 59 & $5,1 \%$ \\
\hline 2005 & 45 & 33 & 3 & 4 & 0 & 0 & 71 & 78 & $6,8 \%$ \\
\hline 2006 & 66 & 32 & 2 & 14 & 8 & 1 & 73 & 98 & $8,5 \%$ \\
\hline 2007 & 53 & 18 & 2 & 21 & 5 & 1 & 42 & 71 & $6,1 \%$ \\
\hline 2008 & 52 & 37 & 0 & 24 & 7 & 1 & 57 & 89 & $7,7 \%$ \\
\hline 2009 & 41 & 26 & 1 & 22 & 3 & 0 & 41 & 67 & $5,8 \%$ \\
\hline 2010 & 47 & 29 & 2 & 17 & 8 & 1 & 48 & 76 & $6,6 \%$ \\
\hline 2011 & 53 & 36 & 2 & 18 & 9 & 1 & 59 & 89 & $7,7 \%$ \\
\hline 2012 & 52 & 25 & 4 & 11 & 9 & 1 & 52 & 77 & $6,7 \%$ \\
\hline 2013 & 36 & 25 & 2 & 10 & 14 & 2 & 33 & 61 & $5,3 \%$ \\
\hline 2014 & 40 & 25 & 2 & 13 & 7 & 2 & 41 & 65 & $5,6 \%$ \\
\hline 2015 & 29 & 23 & 5 & 6 & 5 & 0 & 36 & 52 & $4,5 \%$ \\
\hline 2016 & 40 & 18 & 0 & 10 & 3 & 0 & 45 & 58 & $5,0 \%$ \\
\hline Total & 725 & 421 & 189 & 172 & 81 & 14 & 690 & 1146 & - \\
\hline Média & 45,31 & 26,31 & 11,8 & 10,75 & 5,1 & 0,87 & 43,12 & 71,6 & - \\
\hline Porcentagem & $63,2 \%$ & $36,7 \%$ & $16,5 \%$ & $15 \%$ & $7 \%$ & $1,2 \%$ & $60,2 \%$ & - & - \\
\hline \multirow[t]{2}{*}{ Valor de $p$} & \multicolumn{2}{|c|}{$<.0001$} & \multicolumn{5}{|c|}{$<.0001$} & & \\
\hline & \multicolumn{4}{|c|}{$2001 \sim 2010$} & \multicolumn{5}{|c|}{$2011 \sim 2016$} \\
\hline Média das décadas & \multicolumn{4}{|c|}{71,6 casos por ano } & \multicolumn{5}{|c|}{67 casos por ano } \\
\hline
\end{tabular}

Fonte: Silva PH, et al., 2016. 
Revista Eletrônica Acervo Saúde / Electronic Journal Collection Health ～ISSN 2178-2091

Tabela 2 - Distribuição dos casos de Tuberculose em um município do estado do Piauí classificados por Zona de Residência e Faixa Etária

\begin{tabular}{|c|c|c|c|c|c|c|c|c|c|c|c|c|c|c|}
\hline \multirow[b]{2}{*}{ Variáveis } & \multicolumn{4}{|c|}{ Zona de Residência } & \multicolumn{10}{|c|}{ Faixa etária } \\
\hline & $\begin{array}{c}\text { Ignorado/Em } \\
\text { Branco }\end{array}$ & Urbana & Rural & Periurbana & $\begin{array}{c}<1 \\
\text { ano }\end{array}$ & $\begin{array}{l}1 \text { a } 4 \\
\text { anos }\end{array}$ & $\begin{array}{l}5 \mathrm{~s} 9 \\
\text { anos }\end{array}$ & $\begin{array}{c}10 \mathrm{a} \\
14 \\
\text { anos }\end{array}$ & $\begin{array}{c}15 \text { a } \\
19 \\
\text { anos }\end{array}$ & $\begin{array}{c}20 \text { a } \\
34 \\
\text { anos }\end{array}$ & $\begin{array}{c}35 \text { a } \\
49 \\
\text { anos }\end{array}$ & $\begin{array}{c}50 \text { a } \\
64 \\
\text { anos }\end{array}$ & $\begin{array}{c}65 \mathrm{a} \\
79 \\
\text { anos }\end{array}$ & $\begin{array}{l}80 \text { anos } \\
\text { ou mais }\end{array}$ \\
\hline 2001 & 2 & 67 & 5 & 0 & - & - & - & - & - & - & - & - & - & - \\
\hline 2002 & 2 & 56 & 2 & 0 & 0 & 0 & 0 & 1 & 5 & 26 & 13 & 11 & 3 & 1 \\
\hline 2003 & 0 & 65 & 7 & 0 & 2 & 0 & 0 & 1 & 4 & 23 & 14 & 12 & 12 & 4 \\
\hline 2004 & 0 & 56 & 3 & 0 & 2 & 0 & 0 & 0 & 1 & 16 & 21 & 16 & 3 & 0 \\
\hline 2005 & 0 & 67 & 11 & 0 & 0 & 0 & 1 & 3 & 6 & 17 & 17 & 22 & 10 & 2 \\
\hline 2006 & 1 & 88 & 7 & 2 & 0 & 0 & 1 & 2 & 10 & 30 & 30 & 11 & 11 & 3 \\
\hline 2007 & 1 & 58 & 12 & 0 & 0 & 0 & 0 & 2 & 10 & 23 & 16 & 10 & 9 & 1 \\
\hline 2008 & 2 & 78 & 8 & 1 & 1 & 1 & 0 & 3 & 6 & 29 & 19 & 16 & 12 & 2 \\
\hline 2009 & 0 & 59 & 8 & 0 & 0 & 0 & 0 & 1 & 4 & 25 & 15 & 14 & 7 & 1 \\
\hline 2010 & 3 & 63 & 10 & 0 & 0 & 0 & 0 & 1 & 7 & 23 & 17 & 16 & 10 & 2 \\
\hline 2011 & 1 & 76 & 11 & 1 & 0 & 2 & 0 & 1 & 5 & 27 & 26 & 18 & 8 & 2 \\
\hline 2012 & 1 & 68 & 8 & 0 & 0 & 0 & 1 & 2 & 5 & 25 & 17 & 16 & 9 & 2 \\
\hline 2013 & 3 & 56 & 2 & 0 & 0 & 1 & 1 & 0 & 3 & 15 & 20 & 9 & 8 & 4 \\
\hline 2014 & 1 & 56 & 8 & 0 & 0 & 0 & 1 & 1 & 6 & 19 & 13 & 15 & 7 & 3 \\
\hline 2015 & 1 & 46 & 5 & 0 & 0 & 0 & 2 & 0 & 4 & 15 & 14 & 10 & 7 & 0 \\
\hline 2016 & 0 & 54 & 4 & 0 & 0 & 0 & 0 & 1 & 2 & 16 & 15 & 18 & 4 & 2 \\
\hline Total & 18 & 1013 & 111 & 4 & 5 & 4 & 7 & 19 & 78 & 329 & 267 & 214 & 120 & 29 \\
\hline Média & 1,6 & 63,3 & 6,9 & 0,25 & 0,33 & 0,3 & 0,47 & 1,3 & 5,2 & 21,93 & 17,8 & 14,27 & 8,0 & 1,93 \\
\hline Porcentagem & $1,5 \%$ & $88,4 \%$ & $9,6 \%$ & $0,3 \%$ & $0,4 \%$ & $0,3 \%$ & $0,6 \%$ & $1,6 \%$ & $6,8 \%$ & $28,7 \%$ & $23,3 \%$ & $18,6 \%$ & $10,4 \%$ & $2,5 \%$ \\
\hline Valor de $p$ & & $<.00$ & & & & & & & & $<.0001$ & & & & \\
\hline
\end{tabular}

Fonte: Silva $\mathrm{PH}$, et al., 2016.

REAS/EJCH | Vol.12(5) | e1916 | DOI: https://doi.org/10.25248/reas.e1916.2020 Página 6 de 10 


\section{DISCUSSÃO}

A tuberculose é reconhecidamente uma doença cuja ocorrência é influenciada por fatores sociais. Esse fato torna necessário que haja amparo governamental e a elaboração de políticas de apoio às pessoas doentes em vários aspectos, principalmente garantindo meios para a continuidade dos cuidados em saúde e promovendo ações de combate aos elementos originários do processo de adoecimento (FERREIRA KR, et al., 2018). O presente estudo identificou que as características sociodemográficas de raça, região de moradia, faixa etária e sexo, da população de um município do estado do Piauí no período estudado, estão diretamente ligadas à incidência da TB.

Tomando como base o sexo, diversos estudos realizados no Brasil encontraram achados semelhantes aos observados na cidade estudada. Mendes AM, et al. (2016) realizaram um estudo epidemiológico do estado do Rio Grande do Sul, levantando dados do período compreendido entre 2003 e 2012. Nessa análise, foi encontrada uma taxa de incidência nos indivíduos do sexo masculino de aproximadamente $65 \%$ dos casos analisados. Belchior AS, et al. (2016) encontraram um panorama similar, apontando uma média de $66,7 \%$ de casos em pacientes do sexo masculino, em uma análise feita na cidade de Manaus.

No presente estudo, os indivíduos do sexo masculino representaram aproximadamente $63,2 \%$ do número de novos casos, com uma média de 45,31 casos por ano da doença, diferindo significativamente dos 26,31 casos de tuberculose do sexo feminino na cidade. Essa realidade pode ser justifica principalmente pelo fato de os indivíduos do sexo masculino se exporem mais ao M. tuberculosis, principalmente em suas atividades laborais, e por conta da maior exposição a situações de risco (CECÍLIO HPM, et al., 2018; ZANOTI MDU, et al., 2011).

Em alguns anos dentro do período analisado, entretanto, observou-se uma tendência à aproximação da incidência de TB em homens e mulheres. Esse fator pode estar relacionado principalmente à redução da divisão das funções laborais entre homens e mulheres, decorrentes da revolução do mercado de trabalho que vêm acontecendo nos últimos anos. Esse processo é caracterizado principalmente pela inserção cada vez maior das mulheres no universo do trabalho regulamentado. Essa maior aproximação nos cenários de convivência por homens e mulheres, expõe ambos os sexos a condições de vulnerabilidade muito semelhantes, em relação à infecção pelo bacilo da tuberculose, e justificaria a aproximação numérica quanto a incidência da doença nos últimos anos do período estudado, o que pode ser visualizado na Tabela 1 (LELIS CT, et al. 2012).

No presente estudo, a distribuição da incidência da tuberculose por raças demonstrou uma diferença mais acentuada para a ocorrência da doença em indivíduos de cor parda, representando $72 \%$ dos casos notificados de TB, com média de 45,31 casos por ano, no período de estudo. Em indivíduos de cor da pele branca a média foi de apenas 10,75 casos anuais; as pessoas com a cor de pele preta apresentaram 5,1 casos anuais; e; por fim; os indivíduos de cor de pela amarela apresentaram uma média de 0,87 casos anuais.

Alguns estudos presentes na literatura analisaram o mesmo panorama, apresentando, entretanto, descrições divergentes em alguns pontos, ao considerar o fator raça e cor de pele. Vale ressaltar que maioria desses estudos encontrados para análise levaram em consideração também as populações indígenas habitantes das regiões analisadas. Os autores Basta PC, et al. (2013) e Ferraz AF e Valente JG (2014) realizaram estudos epidemiológicos no estado do Mato Grosso do Sul em que foram analisados os dados referentes à ocorrência de tuberculose em indígenas e não indígenas, segundo raça/cor. Segundo tais pesquisas, no período de 2001 a 2009, a incidência média foi de 209,0 para indígenas, e 22,4 para pardos. Belchior AS, et al. (2016), entretanto, ao analisar a situação epidemiológica da tuberculose na cidade de Manaus no ano de 2011, observaram uma incidência de 67,9\% em pessoas de cor parda.

As populações indígenas, que muitas vezes vivem em situação de extrema pobreza, estão expostas a condições que aumentam o risco de transmissão da TB. Esses indivíduos estão mais suscetíveis a desnutrição, fator que deprime o mecanismo de imunidade celular e facilita a infecção pelo bacilo, e a condições precárias de moradia, com alta densidade demográfica intradomiciliar e falta de ventilação, o que possibilita uma maior exposição à bactéria. Todos esses fatores justificariam a maior incidência da tuberculose 
em indígenas (FERRAZ AF e VALENTE JG, 2014; MARQUES AMC e CUNHA RV, 2003). Essa realidade de desnutrição, falta de serviços básicos de saneamento e alta densidade demográfica, entretanto, não é exclusividade das populações indígenas. A cidade, possui, em toda sua extensão, apenas $23,5 \%$ de rede de saneamento básico adequada, e uma densidade demográfica de 334,51 pessoas $/ \mathrm{km}^{2}$ (IBGE, 2019). A população de cor parda, no município, totaliza 82.987 pessoas, representando aproximadamente $57,1 \%$ da população (IBGE, 2019). Desse modo, a alta densidade demográfica do município e a precária cobertura de serviços sanitários para a população, podem ser os principais fatores condicionantes das altas taxas de incidência de tuberculose nas populações de cor parda, realidade que pode ser visualizada na tabela 1. Segundo MARQUES AMC e CUNHA RV (2003), em todo o mundo, o mapa da pobreza extrema se adapta perfeitamente ao mapa da tuberculose.

Como discutido até então, as condições de vida da população e sua situação socioeconômica são condições que influenciam diretamente a taxa de incidência de tuberculose. Esses fatores, entretanto, não são totalmente uniformes e sofrem variações dentro de um mesmo município, levando, desse modo, a variações também quanto a incidência da TB ao se tomar por base as características do espaço geográfico em que estão inseridos cada indivíduo. Desse modo, o presente estudo encontrou, dentro da cidade, um cenário de distribuição epidemiológica semelhante ao observado em outros estudos. Santos TA e Martins MMF (2018) realizaram um estudo transversal utilizando dados do Sistema de Informação de Agravos de Notificação para analisar a situação epidemiológica da TB no estado do Rio de Janeiro. Nesse estudo, verificou-se que $98,9 \%$ dos casos notificados da doença eram de moradores das zonas urbanas dos municípios.

Os novos casos de tuberculose em moradores da zona urbana representam $89,9 \%$ de todos os casos notificados no município, uma taxa muito superior às zonas rural e periurbana, como pode ser visto na tabela 2. As zonas urbanas são caracterizadas por uma alta concentração populacional, e elevado contato interpessoal. Por ser uma doença cuja transmissão se dá por dissipação de fluidos respiratórios, o contato direto com pessoas doentes e a convivência em espaços limitados é um dos principais fatores de risco para a transmissão do bacilo (KOZAKEVICH GV e SILVA RM, 2015).

A partir dos dados produzidos, foi observado que a ocorrência de novos casos de tuberculose se concentrou principalmente em pessoas com idade entre 20 e 64 anos de idade representando aproximadamente $75 \%$ da incidência. Belchior AS, et al. (2016) encontraram uma situação semelhante ao analisar o surgimento de novos casos de tuberculose na cidade de Manaus no ano de 2011, onde os indivíduos com idade entre 18 e 49 anos representaram 75,8\% da incidência. Coelho DMM, et al. (2010), realizaram um levantamento epidemiológico da tuberculose na cidade de Teresina, onde encontrou-se uma taxa de incidência de 65,64/100.000 na faixa de 20 a 49 anos.

As características socias do próprio ser humano propiciam que com o aumento da idade haja maior exposição e possibilidade de infecção pelo $M$. tuberculosis. Diversos estudos na literatura confirmam a associação entre a idade e a possibilidade de infecção. Essa relação se dá principalmente pelas mudanças de comportamento que acompanham o aumento da idade (GAZETTA CE, et al., 2006). A faixa etária entre 20 e 34 anos, observada no presente estudo como de maior risco à infecção, representa o período da vida considerado economicamente ativo, e em que o indivíduo intensifica suas relações sociais, principalmente relacionadas ao trabalho (SNIDER DE, et al., 1985; REIDER HL, 2003).

\section{CONCLUSÃO}

A análise dos dados do presente estudo sobre a epidemiologia da tuberculose, durante o período de 2001 a 2016, e sua relação com a realidade sociodemográfica da população acometida, permite uma avaliação clara da realidade da doença na região e possibilita o entendimento de que o perfil epidemiológico segue parâmetros comuns a outras regiões. Pessoas do sexo masculino, que habitam as regiões urbanas do município, pessoas de cor parda, indivíduos do sexo masculino, e pessoas com faixa etária entre 20 e 64 anos de idade constituem o grupo que está mais exposto e possui maior probabilidade de infecção pelo bacio da tuberculose. A partir dessa análise, portanto, deve-se dar mais atenção ao perfil da incidência da 
tuberculose no município, formulando ações que visem combater a doença em sua origem e frear seu avanço. Nesse contexto, mostra-se fundamental a aplicação de meios práticos que permitam a notificação da incidência e da prevalência da tuberculose com eficiência. Esse processo é essencial para o controle epidemiológico, avaliação da eficácia dos meios empregados para o tratamento dos doentes e prevenção da transmissão. Somente dessa forma será possível a elaboração de métodos de prevenção ao abandono do tratamento da TB.

\section{REFERÊNCIAS}

1. AZEVEDO MAJ, et al. Redes sociais de usuários portadores de tuberculose: a influência das relações no enfrentamento da doença. Saúde Debate 2018; 42(117):442-454.

2. BASTA PC, et al. Social inequalities and tuberculosis: an analysis by race/color in Mato Grosso do Sul, Brazil. Rev Saúde Pública 2013; 47(5):854-64.

3. BELCHIOR AS, et al. Differences in the Clinical epidemiological profile between new cases of tuberculosis and retreatment cases after default. Rev Esc Enferm USP 2016; 50(4):622-627.

4. CAMPOS HS. Diagnóstico da Tuberculose. Rev Pulmão 2006; 15(2):92-99

5. CARVALHO AL. Informação em saúde como ferramenta estratégica para a qualificação da gestão e o fortalecimento do controle social no SUS. Rev Tempus - Actas de Saúde Coletiva 2009 jul-set; 3(3):16-30.

6. CECÍLIO HPM, et al. Tuberculosis mortality trend in the state of Paraná, Brazil - 1998-2012. Ciência \& Saúde Coletiva2018; 23(1):241-248.

7. COÊLHO DMM, et al. Epidemiological Features of Tuberculosis in the city of Teresina, Piaui state, Brazil, from 1999 to 2005. Epidemiol Serv Saúde 2010 jan-mar; 19(1):33-42.

8. CUNHA EA, et al. A diagnosis of pulmonar tuberculosis and drug resistance among inmates in Mato Grosso do Sul, Brazil. Rev Soc Bras Med Trop 2018 May-June; 51(3):324-330.

9. FERRAZ AF, VALENTE JG. Aspectos epidemiológicos da tuberculose pulmonar em Mato Grosso do Sul. Rev Bras Epidemiol 2014 jan-mar; 255-266.

10. FERREIRA ALS, et al. Costs of the search for tuberculosis diagnosis: impact on the family economy. Rev Cuid 2018; 9(3):2400-12.

11. FERREIRA KR, et al. Representations on adherence to the treatment of Multidrug-Resistant Tuberculosis. Rev Esc Enferm USP 2018; 52: e03412.

12. GAZETTA CE, et al. O controle de comunicantes de tuberculose no programa de controle da tuberculose em um município de médio porte da Região Sudeste do Brasil, em 2002. J Bras Pneumol 2006; 32(6):559-65.

13. INTITUTO BRASILEIRO DE GEOGRAFIA E ESTATíSITCA. Panorama Geral do município de Parnaíba.

14. KOZAKEVICH GV, SILVA RM. Tuberculose: Revisão de literatura. Arq. Catarin Med. 2015 out-dez; 44(4): 34-47.

15. LELIS CT, et al. The women insertion in the çabour market and its implications for the food habits of women and their families. Saúde Debate Rio de Janeiro, 2012 out-dez; 36(95):523-532.

16. MARQUES AMC, CUNHA RV. Assisted treatment and tuberculosis cure and treatment dropout rates in the GuaraníKaiwá Indian nation in the municipality of Dourados, Mato Grosso do Sul, Brazil. Cad Saúde Pública, 2003 set-out; 19(5)1405-1411.

17. MELLO FCQ, et al. Tuberculose: onde estamos? J Bras Pneumol 2018; 44(2):82-82.

18. MENDES AM, et al. Epidemiologic situation of tuberculosis in Rio Grande do Sul: an analysis about Sinan's data between 2003 and 2012 focusing on indigenous peoples. Rev Bras Epidemiol jul-set 2016; 19(3):658-669.

19. MINISTÉRIO DA SAÚDE (BR), Grupo Hospitalar Conceição. Tuberculose na Atenção Primária à Saúde. 2. ed. Porto Alegre: Hospital Nossa Senhora da Conceição, 2013.

20. MINISTÉRIO DA SAÚDE (BR), Secretaria de Atenção à Saúde, Departamento de Atenção Básica. Tuberculose na Atenção Primária à Saúde. 2. ed. Brasília: Ministério da Saúde, 2011.

21. MINISTÉRIO DA SAÚDE (BR), Secretaria de Vigilância em Saúde, Departamento de Vigilância das Doenças Transmissíveis. Manual de Recomendações para o Controle da tuberculose no Brasil. Brasília: Ministério da Saúde, 2018.

22. MINISTÉRIO DA SAÚDE (BR). Secretaria de Vigilância em Saúde, Departamento de Vigilância das Doenças Transmissíveis. Cartilha para o Agente Comunitário de Saúde. 1. ed. Brasília: Ministério da Saúde, 2017

23. OLIVEIRA GP, et al. Mortality information system for identifying underreported cases of tuberculosis in Brazil. Rev Bras Epidemiol 2012; 15(3):468-77.

24. PELISSARI DM, et al. Oferta de serviços pela atenção básica e deteç̧ão da incidência de tuberculose no Brasil. Rev Saude Publica 2018; 52:53. 
25. PEREIRA AG, et al. Análise espacial de casos de tuberculose e associação com fatores socioeconômicos: uma experiência no município do Rio de Janeiro. Cad. Saúde Colet. 2018; 26(2): 203-210.

26. PROCÓPIO MJ. Controle da tuberculose: Uma proposta de integração ensino-serviço. 7. ed. Rio de Janeiro: Editora FIOCRUZ, 2014.

27. RABAHI MF. Tuberculose e Tabagismo. Pulmão 2012; 21(1):46-49.

28. REIDER HL. Contacts of tuberculosis patients in highincidence countries. Int J Tuberc Lung Dis. 2003;7(12 Suppl 3):S333-6.

29. RODRIGUES FF, et al. Avaliação dos serviços farmacêuticos na Atenção Primária à Saúde no cuidado ao paciente com tuberculose. Saúde Debate 2018 out; 42(2):173-187.

30. SANTOS JN, et al. Fatores associados à cura no tratamento da tuberculose no estado do Rio de Janeiro, 2011 2014*. Epidemiol Serv Saúde 2018; 27(3)e2017464.

31. SANTOS TA, MARTINS MMF. Perfil dos casos de reingresso após abandono do tratamento da tuberculose em Salvados, Bahia, Brasil. Cad Saúde Colet 2018; 26(3)233-240.

32. SAS. Institute SAS (Statistical Analysis System). User's Guide. Cary NC: SAS Institute Inc. 2003. 129p.

33. SILVA DR, et al. Global TB Network: trabalhando juntos para eliminar a tuberculose. J Bras Pneumol 2018; 44(5):347349.

34. SILVA MEN, et al. General aspects of tuberculosis: an update on the etiologic agent and treatment. RBAC 2018; 50(3):228-32.

35. SISTEMA IBGE. Tabela de correlação da população residente por cor ou raça.

36. SNIDER DE, et al. Infection and disease among contacts of tuberculosis cases with drug-resistant and drugsusceptible bacilli. Am Rev Respir Dis. 1985; 132(1):125-32.

37. ZANOTI MDU, et al. Epidemiological profile of women hospitalized for tuberculosis in a specialized hospital (20052009). Cuidarte Enfermagem 2011 jul-dez; 5(2):104-108. 